\title{
Response of an annular electrostatic probe for a right cylindrical spacer
}

\author{
Johansson, Torben; MacAllister, I. W.
}

Published in:

Annual Report Conference on Electrical Insulation and Dielectric Phenomena, 2002

Link to article, DOI:

10.1109/CEIDP.2002.1048749

Publication date:

2002

Document Version

Publisher's PDF, also known as Version of record

Link back to DTU Orbit

Citation (APA):

Johansson, T., \& MacAllister, I. W. (2002). Response of an annular electrostatic probe for a right cylindrical spacer. In Annual Report Conference on Electrical Insulation and Dielectric Phenomena, 2002 IEEE.

https://doi.org/10.1109/CEIDP.2002.1048749

\section{General rights}

Copyright and moral rights for the publications made accessible in the public portal are retained by the authors and/or other copyright owners and it is a condition of accessing publications that users recognise and abide by the legal requirements associated with these rights.

- Users may download and print one copy of any publication from the public portal for the purpose of private study or research.

- You may not further distribute the material or use it for any profit-making activity or commercial gain

- You may freely distribute the URL identifying the publication in the public portal

If you believe that this document breaches copyright please contact us providing details, and we will remove access to the work immediately and investigate your claim. 


\title{
2002 Annual Report Conference on Electrical Insulation and Dielectric Phenomena
}

\section{Response of an annular electrostatic probe for a right cylindrical spacer}

\author{
T. Johansson and I. W. McAllister \\ Department of Electric Power Engineering \\ Technical University of Denmark \\ Lyngby, Denmark
}

\begin{abstract}
The response of an annular electrostatic probe mounted in an electrode is examined with reference to a right cylindrical spacer. The study involves using the probe $\lambda$ function to derive characteristic parameters. These parameters enable the response of the probe to different charge distributions to be quantitatively assessed.
\end{abstract}

\section{Introduction}

Bound annular electrostatic probes have recently been employed to observe the changes in electric field due to the accumulation of surface charge on a right cylindrical spacer in vacuum [1-3]. The word bound signifies that the probe is mounted in the electrode in contact with the solid dielectric, see Figure 1. Thus, as the probe is an integral part of this electrode, such probes lack the freedom of movement necessary to implement a scanning procedure which is required for a correct quantitative evaluation of the surface charge to be made [4-6].

In [1-3], the relation between the probe signal $V_{p}$ and the probe field strength $E_{\mathrm{p}}$ is given as

$$
E_{\mathrm{p}}=\frac{C V_{\mathrm{p}}}{S \varepsilon}
$$

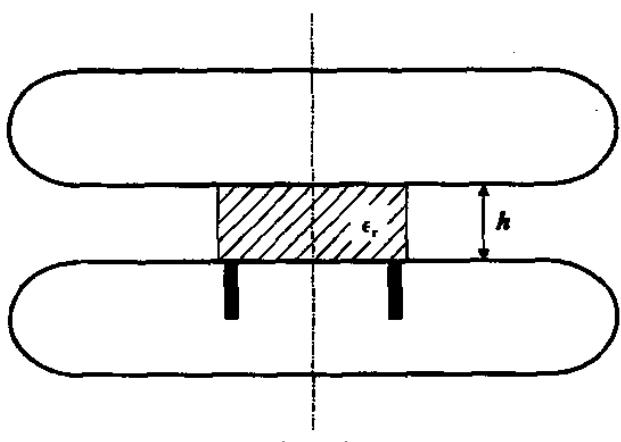

Figure 1: Bound probe/dielectric spacer geometry. where $C$ represents the measuring capacitor, $S$ the surface area of the probe, and $\varepsilon$ the permittivity of the dielectric spacer. This expression implies also that the surface charge density $\sigma_{p}$ at the probe is assumed to be constant, i.e. $\sigma_{p}=C V_{p} / S$.

If the capacitive component of $V_{\mathrm{p}}$ associated with the applied voltage is neglected, then the product $C V_{p}$ effectively represents the probe induced charge $q$. Thus with respect to the field component $E_{\mathrm{ps}}$ associated with the surface charge, we have

$$
E_{\mathrm{ps}}=\frac{q}{S \varepsilon}
$$

i.e. the electric field recorded using a probe is in effect a measurement of the charge induced on the probe by the surface charge.

The relationship between the induced charge and the source charge is expressible in terms of a general function, the $\lambda$ function [7]. Using this function it is possible to examine the response of electrostatic probes. In the present study, the response of annular probes to surface charge on the side of a right cylindrical spacer is investigated, and discussed with respect to experimental studies reported in the literature.

\section{The $\lambda$ function}

The $\lambda$ function is the proportionality factor between the charge induced on the probe to any charge within the dielectric volume or at an interface [7]. If it is assumed that the volume charge density in the solid dielectric is zero, then this relationship can be expressed simply as

$$
q=-\iint_{A_{0}} \lambda \sigma \mathrm{d} A
$$


where $q$ is the Poissonian induced charge on the probe sensor plate, and $\sigma$ is the surface charge density on the surface element $\mathrm{d} A$ of $A_{\mathrm{o}}$, the surface of the solid dielectric.

The dimensionless parameter $\lambda$ is a solution of the general Laplace equation for the complete system geometry:

$$
\vec{\nabla} \bullet(\varepsilon \vec{\nabla} \lambda)=0
$$

The boundary conditions are $\lambda=1$ at the probe sensor plate and $\lambda=0$ at all other electrodes. In addition, at dielectric interfaces the normal derivatives of $\lambda$ must obey the condition

$$
\varepsilon_{+}\left[\frac{\partial \lambda}{\partial n}\right]_{+}=\varepsilon_{-}\left[\frac{\partial \lambda}{\partial n}\right]_{-}
$$

where the + and - signs refer to the opposite sides of the interface. As (4) is just Laplace's equation, any standard method of solving this equation can be employed to evaluate the variation of $\lambda$ along the interface. On this occasion, solutions of Laplace's equation were obtained using a finite element software package. These solutions were then used to study the dependence of the probe response upon the surface charge distribution.

\section{Probe/dielectric geometry}

The essential geometry of the annular probe used in this study is shown in Figure 2, in which $d_{\mathrm{i}}$ and $d_{\mathrm{o}}$ are the inner and outer diameters of the annular probe sensor plate, while $w$ represents the width of this sensor, i.e. $w=d_{0}-d_{\mathrm{i}}$. The gap between the sensor plate and the remainder of the electrode is $\Delta$.

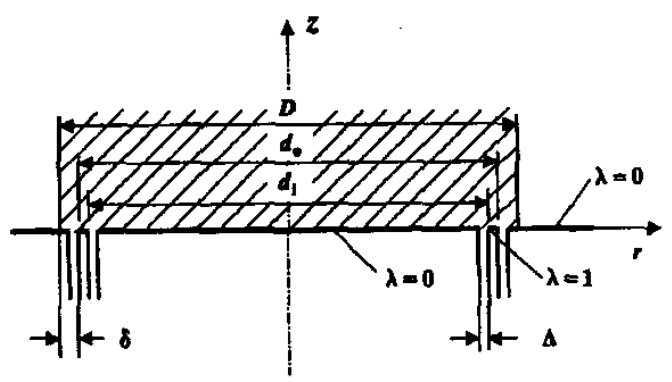

Figure 2: Geometric detail of bound probe.
The cylindrical dielectric, of relative permittivity $\varepsilon_{\mathrm{r}}$, has an overall diameter of $D$ and a height $h$ in a direction normal to the plane electrodes, which are grounded, while the gap $\delta$ represents the amount by which the dielectric overlaps the probe, i.e. $2 \delta=$ $D-d_{0}$.

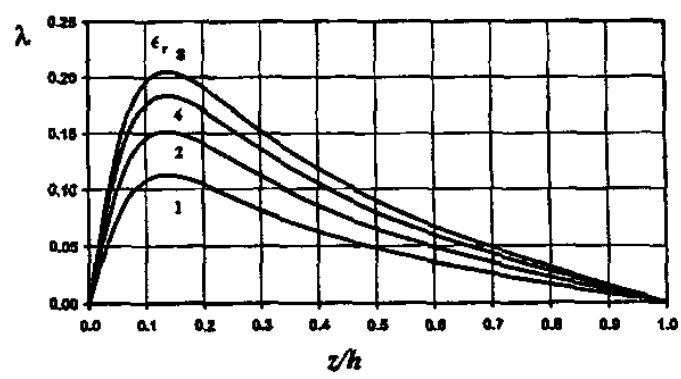

Figure 3: Variation of $\lambda$ along cylindrical interface for $\delta / d_{\mathrm{p}}=0.02$.

If $d_{\mathrm{p}}$ represents the mean diameter of the probe, i.e., $2 d_{\mathrm{p}}=d_{\mathrm{o}}+d_{\mathrm{i}}$, then the probe/dielectric geometry can be defined in terms of the ratios $D / d_{\mathrm{p}}, h / d_{\mathrm{p}}$, $\delta / d_{\mathrm{p}}, w / d_{\mathrm{p}}$, and $\Delta / w$. For the present study the following parameter values were used: $D / d_{\mathrm{p}}=1.06$, $h / d_{\mathrm{p}}=0.2, \delta / d_{\mathrm{p}}=0.02, w / d_{\mathrm{p}}=0.04$, and $\Delta / w=$ 0.05 . These normalized dimensions correspond essentially with those employed in [1-3]. In the direction normal to the probe/gap axis, the extent of the plane electrodes is much greater that the probe lateral dimensions.

The variation of the $\lambda$ along the cylindrical dielectric interface from cathode to anode $(0 \leq z / h \leq 1)$ is shown in Figure 3 for $\delta / d_{\mathrm{p}}=0.02$; i.e. if $d_{\mathrm{p}}=50 \mathrm{~mm}$, then $\delta=1 \mathrm{~mm}$ as in [1-3], and for various values of $\varepsilon_{\mathrm{r}}$. From this Figure, it is evident that $\lambda$ displays a maximum value $\left(\lambda_{\max }\right)$ for $z h \cong 1.35$, effectively independent of $\varepsilon_{\mathrm{r}}$, and that this value increases with increasing values of $\varepsilon_{\mathrm{r}}$. As $\lambda=0$ for $z / h=0$ and $z / h=$ 1 , the form of the $\lambda$ distribution is not unexpected. Moreover, as the dielectric interface of interest is normal to, and physically offset from the probe surface, the values of $\lambda_{\max }$ are less than those reported for other bound probed situations, see [8]. This implies that, for the present probe/interface arrangement, the probe is less sensitive than the usual parallel probe/interface situation. 


\section{Surface charge distributions}

To study the influence of the surface charge on the probe response, the response is examined for several charge distributions. On this occasion the following distributions were used

$$
\begin{aligned}
& \sigma=\sigma_{0} \\
& \sigma=\sigma_{1}\left(1-\frac{z}{h}\right) \\
& \sigma=\sigma_{2} \exp (-5 z / h)
\end{aligned}
$$

for $0 \leq z \leq h$, or more generally

$$
\sigma=\sigma_{n} f_{n}(z / h)
$$

with $n=0,1,2$. As $f_{n}(0)=1$ and $f_{n}(1) \ll 1, \sigma_{n}$ represents the maximum value of $\sigma$.

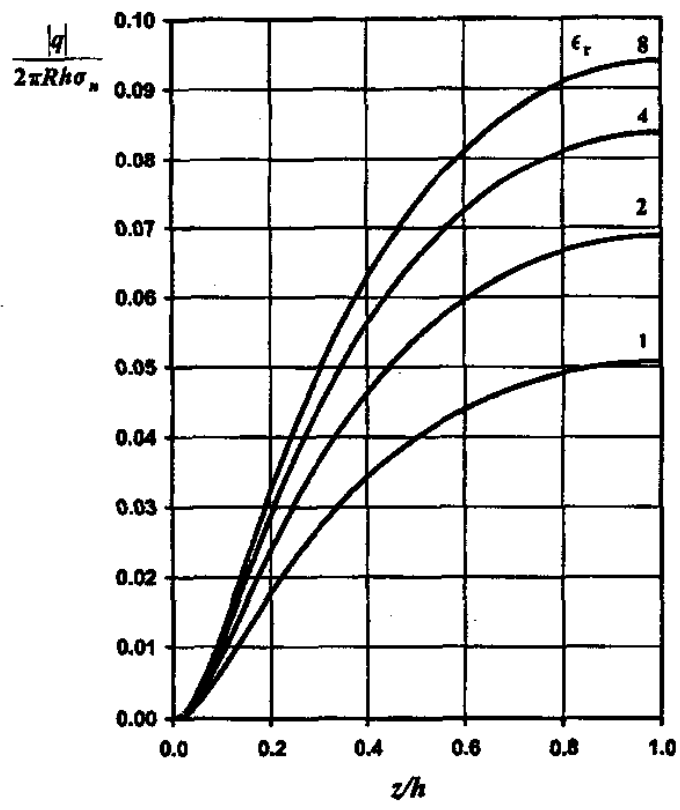

Figure 4: Variation of $|q|$ for $\sigma=\sigma_{0}$

If $r, z$ represent cylindrical coordinates, see Figure 2, then for a cylindrical surface charge located at the insulator surface $(r=R)$, the charge $q$ induced on the sensor plate is given by

$$
q(z)=-2 \pi R \sigma_{n} \int_{0}^{z} \lambda\left(R, z^{\prime}\right) f_{n}\left(z^{\prime}\right) \mathrm{d} z^{\prime}
$$

where $z^{\prime}$ represents a dummy variable, and $R(=D / 2)$ is the radius of the cylindrical dielectric. The actual charge $Q$ accumulating on the insulator surface is

$$
Q=2 \pi R \sigma_{n} \int_{0}^{z} f_{n}\left(z^{\prime}\right) \mathrm{d} z^{\prime}
$$

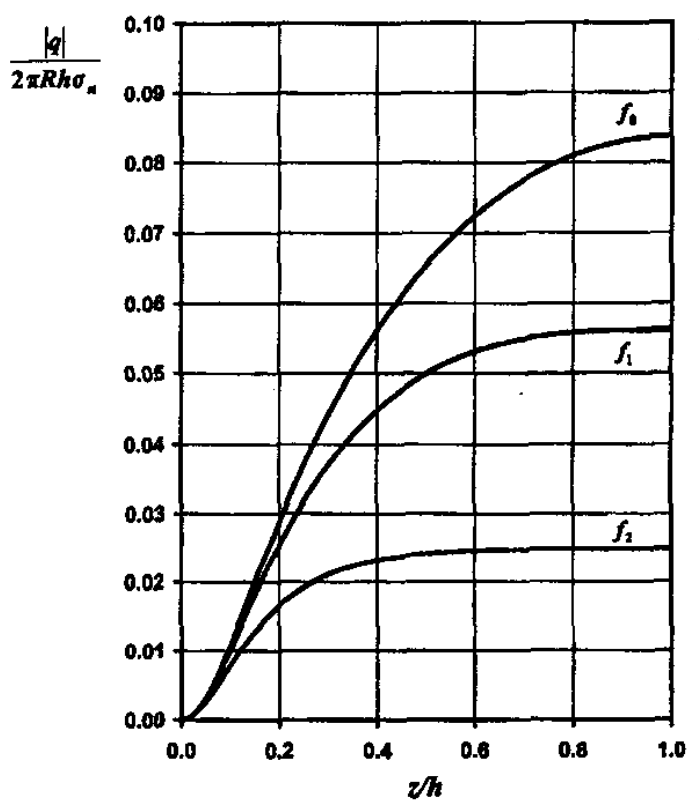

Figure 5: Variation of $|q|$ for different $\sigma$ distributions: $\varepsilon_{r}=4$.

This expression can be readily evaluated for the $\sigma$ distributions discussed above.

For $\sigma=\sigma_{0}$, the influence of $\lambda$ upon the induced charge is illustrated in Figure 4 for various $\varepsilon_{\mathrm{r}}$. Owing to the nature of the $\lambda$ distribution: i.e. the two $\lambda=0$ boundaries create a $\lambda$ distribution of finite spatial extent, it is observed from Figure 4 that the maximum value of the induced charge $\left(q_{\max }\right)$ is effectively proportional to $\lambda_{\max }$. Such a situation does not arise with the usual bell-shaped $\lambda$ distribution, see [8] . Similar induced charge behaviour is displayed with the two other $\sigma$ distributions.

The variation of the induced charge with the spatial extent of the surface charge is illustrated in Figure 5 for 
the three charge distributions, and $\varepsilon_{\mathrm{r}}=4$. In Figure 5, the $|q|$ curves have been normalized to a common $\sigma_{n}$, i.e. $\sigma_{0}=\sigma_{1}=\sigma_{2}$. This implies that each distribution is associated with a different total charge $Q_{\mathrm{T}}$. A common $Q_{\mathrm{T}}$, i.e. $Q_{\mathrm{T} 0}=Q_{\mathrm{T} 1}=Q_{\mathrm{T} 2}$, leads to another relation between $\sigma_{0}, \sigma_{1} \& \sigma_{2}$. For the present situation, we have $Q_{\mathrm{T} 0}>Q_{\mathrm{T} 1}>Q_{\mathrm{T} 2}$ and thus the greatest value of $q_{\max }$ will be associated with $f_{0}$, while the smallest $q_{\max }$ will be related to $f_{2}$.

However from Figure 5, it is evident that, even for 3 widely different $\sigma$ distributions, the form of each $|q|$ variation is similar. Such behaviour is associated with the integral nature of the induced charge phenomenon. The non-uniformity of the $\sigma$ distribution affects how rapidly the induced charge attains its maximum value.

\section{Discussion}

We have examined the response of an annular probe with respect to the usual method of performing probe measurements: i.e., all electrodes, with the exception of the sensor electrode, are earthed. This condition is introduced to circumvent the fact that the source charge induces a charge on all electrodes. Those electrodes not held at zero potential, will undergo a change in potential. Due to the inherent partial capacitance associated with each electrode-pair, such changes in potential will be registered at the sensor electrode.

This latter situation will arise in the studies of Yamamoto et al. [1-3], in which a voltage is applied to the anode to promote the discharge development during the probe measurements. Consequently, the interpretation of these measurements will be anything but straightforward. In addition, as the measurements reported are real-time observations of surface charging, the probe is responding simultaneously to both the spatial and temporal variation of the surface charge. This situation implies that the interpretation of such measurements is virtually impossible without some auxiliary information about the discharge development.

In [1-3], the authors undertook a discharge simulation based on the concept of the secondary electron emission avalanche. Apart from deriving surface charge distributions, the authors' results indicate that the associated probe field strength is nonuniform. The degree of non-uniformity is dependent on the magnitude and spatial extent of the surface charge. However the authors do not discuss the corresponding temporal response of the probe with respect to the spatial and temporal features of the discharge model.
Consequently, the above aspects preclude a proper discussion of these experimental probe measurements in relation to the present study.

\section{Conclusion}

From an examination of the response of an annular probe to various surface charge distributions, it is evident that the form of the surface charge distribution is not readily discernible. This behaviour is related to the integral nature of the probe response.

In addition, owing to the geometric disparity between the probe and the dielectric interface, the values of the relevant $\lambda$ function are considerably smaller than those reported previously for other probe arrangements. Consequently for the present probe/interface geometry, the sensitivity of the probe is low.

\section{Acknowledgment}

The authors wish to thank J. Berril for producing both this WORD document and the associated PDF file.

\section{References}

[1] O. Yamamoto, T. Takuma and Y. Tanabe, "Real-time observation of surface charging on a cylindrical insulator in vacuum", IEEE Trans. Diel. Elect. Insul., Vol.5, pp.961-965, 1998.

[2] O. Yamamoto, T. Takuma, Y. Kakehashi, S. Ikoma and T. Shioiri, "Delay characteristics of surface charging on a cylindrical insulator in vacuum", IEEE Trans. Diel. Elect. Insul., Vol.7, pp.812-817, 2000.

[3] O. Yamamoto and T. Takuma, "Influence of surface roughness on flashover and charging of a cylindrical insulator in vacuum", 12th International Symposium on High Voltage Engineering, Bangalore, pp.469-472, 2001.

[4] M. Yashima, H. Fujinami and T. Takuma, "Measurement of accumulated charge on dielectric surfaces with an electrostatic probe", in L. G. Christophorou and D. W. Bouldin (eds.), Gaseous Dielectrics V. Pergamon New York, pp.242-247, 1987.

[5] T. O. Rerup, G. C. Crichton and I. W. McAllister, "Using the $\lambda$ function to evaluate probe measurements of charged dielectric surfaces", IEEE Trans. Diel. Elect. Insul., Vol. 3, pp.770-777, 1996.

[6] T. Takuma, M. Yashima and T. Kawamoto, "Principle of surface charge measurement for thick insulating specimens", IEEE Trans. Diel. Elect. Insul., Vol.5, pp.497-504, 1998.

[7] A. Pedersen, "On the electrostatics of probe measurements of surface charge densities", in L. G. Christophorou and D. W. Bouldin (eds.), Gaseous Dielectrics V, Pergamon New York, pp.235-240, 1987.

[8] T. Johansson, G. C. Crichton and I. W. McAllister, "Measurement of surface charge densities associated with surface discharges", XIII Intemational Conference on Gas Discharges and their Applications, Glasgow, pp.892-895, 2000. 